\title{
A forward branching phase space generator for hadron colliders
}

\author{
Terrance M. Figy ${ }^{a}$ and Walter T. Giele ${ }^{b}$ \\ ${ }^{a}$ Department of Mathematics, Statistics, and Physics, Wichita State University, \\ 1845 Fairmount Street, Wichita, KS 67260, U.S.A. \\ ${ }^{b}$ Theory Group, Fermilab, \\ P.O. Box 500, Batavia, IL 60510, U.S.A.
}

E-mail: terrance.figy@wichita.edu, giele@fnal.gov

ABSTRACT: In this paper we develop a projective phase space generator appropriate for hadron collider geometry. The generator integrates over bremsstrahlung events which project back to a single, fixed Born event. The projection is dictated by the experimental jet algorithm allowing for the forward branching phase space generator to integrate out the jet masses and initial state radiation. When integrating over the virtual and bremsstrahlung amplitudes this results in a single $K$-factor, assigning an event probability to each Born event. This $K$-factor is calculable as a perturbative expansion in the strong coupling constant.

One can build observables from the Born kinematics, giving identical results to traditional observables as long as the observable does not depend on the infrared sensitive jet mass or initial state radiation.

KeYwords: Jets, NLO Computations

ArXiv EPrint: 1806.09678 


\section{Contents}

1 Introduction 1

2 The forward branching phase space generator 3

2.1 The Born phase space generator 5

2.2 The final state FBPS generator 6

$\begin{array}{lll}2.3 & \text { The initial state FBPS generator } & 7\end{array}$

$\begin{array}{lll}2.4 \text { Kinematics } & 10\end{array}$

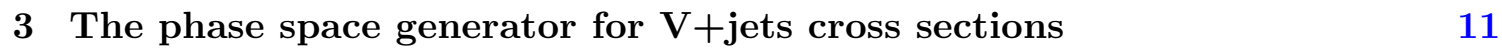

$\begin{array}{lll}4 & \text { Conclusions } & 13\end{array}$

\section{Introduction}

Great advances have been made over the last decades in calculating scattering amplitudes contributing to higher order corrections for processes including jets. In contrast the Monte Carlo methods of integrating over phase space have not evolved much, relying instead on advances in computer power. Given this, there was no urgency to develop improved phase space integration methods. However, the required precision of predictions needed for current and future experimental analyses makes the development of improved phase space integration methods a crucial task. In this paper, we will investigate the promising avenue of projective phase space generators [1-8].

The basics of Monte Carlo integration are simple. Take as an example the process $p p \rightarrow V+1$ jet at Next-to-Leading (NLO) order. One generates random phase space events for the $V+1$ massless particle production, assisted by importance sampling as implemented in, for example, VEGAS [9], and evaluates the Born and dressed Virtual amplitudes to obtain the virtual weight. The observable under study is calculated from the momenta and the weight is binned accordingly in a histogram. This procedure is repeated for the $V+2$ massless particle production. The events are randomly generated, used to evaluate the regulated bremsstrahlung amplitude, a jet algorithm is applied and the observable calculated. Finally, the bremsstrahlung amplitude weight is added to the corresponding bin in the histogram.

In this procedure the use of finite width bins is essential to ensure the cancellations between the virtual and bremsstrahlung contributions, needed to obtain a physical prediction. There are many drawbacks to this method. First of all, the bin edges can cause issues in the cancellations leading to instabilities which might need to be regulated. Secondly, the higher order phase space is extended with respect to the Born phase space. Consequently higher order corrections for an observable cover kinematic regions not accessible to the 
Born results, leading to instabilities in the predictions. Finally, in contrast to Leading Order (LO), one cannot define a weight at higher orders to a single event [10-13]. At LO each $V+1$ jet event has a probabilistic weight associated with it, allowing the events to be unweighted. This is an important step as the subsequent showering and detector simulations are potentially time expensive. At NLO and beyond this is no longer possible, making higher order predictions far more cumbersome and time consuming [14, 15].

To alleviate these issues and obtain more efficient Monte Carlo integration methods for higher order calculations one has to change the basic method of phase space integration. A single $p p \rightarrow V+$ jet(s) event is a well defined multi-dimensional observable with respect to the reconstructed final state object. A scattering probability should be calculable orderby-order in the perturbative expansion. This single observed event is semi-inclusive with respect to the underlying partonic event due to the use of a jet algorithm necessary to obtain the jet final state. We have to integrate the partonic bremsstrahlung events over the jet cones and over the initial state radiation not associated with jets under the constraint of a fixed $V+$ jet(s) event. In doing this one will obtain an infra-red stable, physical prediction order-by-order in perturbation theory. Such a method would allow for the generation of unweighted higher order events, possess no phase space extensions and possess no other sources of instabilities in the predictions. In other words, all the artificial instabilities introduced by the integration method and observable definitions are removed. In two previous papers the methodology of Forward Branching Phase Space (FBPS) generators was developed to some extent. The first paper [1] develops the principles of the method from a more theoretical perspective. It uses a $3 \rightarrow 2$ jet clustering algorithm instead of a $2 \rightarrow 1$ clustering algorithm used in experiments. While from a theoretical point of view the $3 \rightarrow 2$ is greatly preferred and results in a streamlined FBPS generator, it is crucial to extend the method to $2 \rightarrow 1$ clustering jet algorithms so these FBPS generators can be used for predictions of measured observables, In ref. [4] the first step in that direction was taken for lepton collider multi-jet observables using the experimental jet algorithms. Lepton collider observables remove most of the complexities arising in hadron collider jet observables, most importantly the initial state hadronic radiation. In this paper we extend the method to hadron colliders, taking into account jet masses and initial state radiation. The FBPS generator constructed in this paper can be used to make predictions for the LHC experiments using the experimental jet algorithms. Before being able to formulate such an improved method, some issues need to be addressed.

For final state radiation, defined as any partonic bremsstrahlung radiation clustered into a jet, the jet algorithm will construct jet momenta which at higher orders are no longer massless. As a result the massless Born jets cannot be associated with the higher order jets. However, we need to consider the jet event as semi-inclusive as far as the partonic contributions are concerned. In other words we have to integrate over all partonic configurations and consequently, jet masses, resulting in the final state bremsstrahlung contribution to the probabilistic weight of the observed $V+$ jet event. In order to accomplish this, the higher order events with massive jets need to be projected onto the Born event with massless jets. This results in opaque jets without any internal structure as far as perturbative QCD is concerned. For hadron colliders the projection choice is obvious, one leaves the transverse 
momentum of the jet, $\vec{p}_{T}^{\mathrm{JET}}$, and the jet rapidity, $\eta_{\mathrm{JET}}$, invariant under the projection. This means that no predictions can be made which depend on the jet masses, as it is integrated over. Observables are constructed from the Born momenta with higher order corrections changing the event weight but not the event kinematics.

For initial state radiation, defined as partonic bremsstrahlung radiation not associated with the jets, the issue is in principle the same but in practice a bit more subtle. Any event has to observe momentum conservation. That is, summing the momenta of the final state objects together with the momenta of the unclustered radiation gives a perfect momentum balance (modulo detector generated restrictions). To obtain a physical result we need to integrate over the unclustered partonic bremsstrahlung radiation. Many avenues to proceed are possible and in this paper we will consider two options for projecting the event onto to Born event. The first option considers all partonic bremsstrahlung radiation as final state radiation, terminating the jet clustering when a preset number of clusters is reached. In the case of $V+1$ jet all hadronic radiation would be combined in a single cluster. The second option follows the traditional jet cone size approach. The transverse momentum of the unclustered parton(s) is taken into account by adding it back to one of the final state objects. As a result the final state objects are balanced in transverse momentum and hence can be associated with a LO momentum configuration. Naively, one would think this would make the event weight sensitive to the initial state radiation. However, the opposite is true. Not accounting for the initial state radiation into the final state objects lead to instabilities in the perturbative predictions. For example the inclusive Vector Boson transverse momentum distribution is sensitive to the initial state radiation because this radiation is not accounted for [16]. As a result a fixed order prediction of this observable will fail.

With the above methods of projecting the partonic bremsstrahlung events onto the Born phase space, we will factorize in section 2 the bremsstrahlung higher order phase space into the Born phase space times the bremsstrahlung phase space. This adds a single 4-momentum integration per added bremsstrahlung parton. The initial and final state FBPS generators will, in section 3, be combined into a single event generator. While most of the techniques in this paper are applicable to $P P \rightarrow V+$ jets and to a lesser extend $P P \rightarrow$ jets, for the more explicit implementation and examples we restrict ourselves to $P P \rightarrow V+1$ jet. The extension to multiple jets is a straightforward generalization. Finally, the results are summarized in section 4 .

\section{The forward branching phase space generator}

In this section we factorize the bremsstrahlung phase space into a Born phase space times an integral over the bremsstrahlung parton momenta. The Born phase space is defined by a preset projection prescription such as a jet algorithm. By construction, restricting the phase space integration to a single Born phase space point will integrate out the bremsstrahlung contribution to that particular Born event. This enables one to calculate the radiative corrections order-by-order in perturbation theory to any observable constructed from these fixed Born momenta. 
To obtain the factorization of the phase space in a Born phase space times the contribution from the bremsstrahlung, we adopt the Forward Branching Phase Space (FBPS) approach $[1,4]$. This method is firmly based on the methods used in parton showers where one generates new radiation starting from a Born event [17]. We imprint on the branching the projective constraints so the bremsstrahlung integrates over the parton configurations reconstructing to the fixed Born event.

A FBPS generator starts from a Born phase space generator producing the momenta of all observable objects from which observables are constructed. For the process of Vector Boson production plus $n$ jets, $P\left(\hat{p}_{a}\right) P\left(\hat{p}_{b}\right) \rightarrow p_{V}(\hat{Q})+J_{1}\left(\hat{p}_{1}\right)+\cdots+J_{n}\left(\hat{p}_{n}\right)$, the Born phase space is given by

$$
d \Phi\left(\hat{p}_{a} \hat{p}_{b} ; \hat{Q},\{\hat{p}\}_{n}\right)=\left(\prod_{i=1}^{n} \frac{d \hat{p}_{i}}{(2 \pi)^{3}} \delta\left(\hat{p}_{i}^{2}\right)\right) \frac{d \hat{Q}}{(2 \pi)^{3}} \delta\left(\hat{Q}^{2}-M_{V}^{2}\right) \delta\left(\hat{p}_{a}+\hat{p}_{b}-\hat{p}_{1}-\cdots-\hat{p}_{n}-\hat{Q}\right),
$$

where $\hat{p}_{a}$ and $\hat{p}_{b}$ are the incoming parton momenta, $\hat{Q}$ the vector boson momentum, $M_{V}$ the vector boson mass and $\{\hat{p}\}_{n}=\left\{\hat{p}_{1}, \hat{p}_{2} \ldots, \hat{p}_{n}\right\}$ the set of $n$ jet momenta. By generating an additional parton we obtain the bremsstrahlung phase space which will have a generic FBPS form. For a NLO generator this is given by

$$
\begin{aligned}
d \Phi\left(p_{a} p_{b} ; Q,\{p\}_{n+1}\right)= & d \Phi\left(\hat{p}_{a} \hat{p}_{b} ; \hat{Q},\{\hat{p}\}_{n}\right) \\
& \times\left(\frac{d p_{n+1}}{(2 \pi)^{3}} \delta\left(p_{n+1}^{2}\right)\right) J\left(\{\hat{p}\}_{n}, p_{n+1}\right) \times \delta\left(M\left(\{\hat{p}\}_{n} \rightarrow\{p\}_{n+1}\right)\right),
\end{aligned}
$$

where the chosen map generating the bremsstrahlung momenta from the Born momenta and the Jacobian $\left.J\left(\{\hat{p}\}_{n}, p_{n+1}\right\}\right)$ needs to be calculated. The projective jet algorithm is given by the inverse map $M^{-1}\left(\{\hat{p}\}_{n} \leftarrow\{p\}_{n+1}\right)$. A minimum requirement for the map is that $\hat{Q}^{2}=Q^{2}$. Furthermore, it is advantageous to separate initial state radiation from final state radiation. One can iterate this factorization trivially to generate multiple bremsstrahlung particles relevant for beyond NLO calculations.

The first step is to define the cluster map, which is as close to the experimentally used object reconstruction as possible. Any infrared safe jet algorithm will suffice, including the family of $k_{T}$-jet algorithms. For final state radiation a parton branches into two partons generating a jet mass. By keeping the transverse momentum, $\vec{p}_{T}=\left(p_{x}, p_{y}\right)$, and the rapidity, $\eta=\frac{1}{2} \log \left(\frac{E+p_{z}}{E-p_{z}}\right)$, of the jet invariant under the branching one can integrate over the jet mass. Obtaining the massless Born jet momentum is a numerically straightforward procedure. For a jet momentum with mass, we rewrite it in coordinates of mass, transverse momentum and rapidity, set the mass to zero and translate it back in a now massless jet with the same transverse momentum and rapidity: $p_{\mu}=\left(p_{x}, p_{y}, p_{z}, E\right) \rightarrow\left(m_{j}, \vec{p}_{T}, \eta\right) \rightarrow\left(0, \vec{p}_{T}, \eta\right) \rightarrow \hat{p}_{\mu}=\left(\hat{p}_{x}, \hat{p}_{y}, \hat{p}_{z}, \hat{E}\right)$. For the initial state radiation one generates unclustered particles (or particles "clustered with the beam jet") creating a transverse momentum imbalance in the final state object momenta. Here one can choose to absorb the generated unclustered momenta in the vector boson momentum or alternatively keep clustering bremsstrahlung momenta into jets until the required number of remaining clusters is obtained by not defining a jet cone size. Using this type 
of projection allows one to calculate the differential cross sections $d^{(4)} \sigma / d Q\left\{d \hat{\bar{p}}_{T}^{(i)} d \hat{\eta}_{i}\right\}_{i=1}^{n}$ point-by-point in the distribution without the need to introduce histograms. Specifying a single point in the distribution gives an unique Born event with fixed momenta, while higher order corrections add a $K$-factor calculable order-by-order in perturbation theory.

\subsection{The Born phase space generator}

We begin by formulating the Born phase space generator in a convenient manner and introduce the notation and conventions that we will use for the remainder of the paper. In order to be able to constrain the rapidity of the vector boson, $\hat{\eta}_{Q}$, the transverse momenta, $\hat{p}_{T}^{(i)}$, rapidity, $\hat{\eta}_{i}$, and the azimuthal angle, $\hat{\phi}_{i}$, of the jets, we will make these kinematic variables explicit integration variables.

We use the convention that hatted variables are for the Born event and unhatted variables are for the branched bremsstrahlung events. The momenta are parameterized as follows

$$
\begin{aligned}
\hat{p}_{a} & =\frac{1}{2}\left(0,0, \hat{x}_{a}, \hat{x}_{a}\right) \\
\hat{p}_{b} & =\frac{1}{2}\left(0,0,-\hat{x}_{b}, \hat{x}_{b}\right) \\
\hat{p}_{\mu}^{(i)} & =\hat{p}_{T}^{(i)}\left(\sin \hat{\phi}_{i}, \cos \hat{\phi}_{i}, \sinh \hat{\eta}_{i}, \cosh \hat{\eta}_{i}\right) \\
\hat{Q}_{\mu} & =\left(\hat{\vec{q}}_{T}, \hat{\alpha}_{T} \sinh \hat{\eta}_{q}, \hat{\alpha}_{T} \cosh \hat{\eta}_{q}\right) \text { where } \hat{\alpha}_{T}=\sqrt{\hat{q}_{T}^{2}+M_{V}^{2}},
\end{aligned}
$$

where $\hat{p}_{a, b}$ are the incoming particle momenta with parton fractions $x_{a, b}$, the jet momenta are given by $\hat{p}_{\mu}^{(i)}$, and the vector boson momentum is given by $\hat{Q}_{\mu}$. Starting from the Born phase space, eq. (2.1), we perform the following transformations:

$$
\begin{aligned}
\frac{d \hat{p}_{i}}{(2 \pi)^{3}} \delta\left(\hat{p}_{i}^{2}\right)= & \frac{d \hat{\vec{p}}_{i}}{2(2 \pi)^{3} E_{i}}=d \hat{p}_{T}^{(i)} d \hat{\eta}_{i} d \hat{\phi}_{i}\left(\frac{\hat{p}_{T}^{(i)}}{2(2 \pi)^{3}}\right) \\
\frac{d \hat{Q}}{(2 \pi)^{3}} \delta\left(\hat{Q}^{2}-M_{V}^{2}\right)= & \frac{d \hat{\vec{Q}}}{2(2 \pi)^{3} E_{q}}=\frac{d \hat{\vec{q}}_{T} d \hat{\eta}_{q}}{2(2 \pi)^{3}}\left(\text { where we used } d \hat{q}_{z}=\hat{E}_{q} d \hat{\eta}_{q}\right) \\
\delta^{(4)}\left(\hat{p}_{a}+\hat{p}_{b}-\hat{p}_{1}-\cdots-\hat{p}_{n}-\hat{Q}\right)= & \delta^{(2)}\left(\sum_{i=1}^{n} \hat{\vec{p}}_{T}^{(i)}+\hat{\vec{q}}_{T}\right) \\
& \times \delta\left(\frac{1}{2} \sqrt{S}\left(\hat{x}_{a}+\hat{x}_{b}\right)-\sum_{i=1}^{n} \hat{p}_{T}^{(i)} \cosh \hat{\eta}_{i}-\hat{\alpha}_{T} \cosh \hat{\eta}_{q}\right) \\
& \times \delta\left(\frac{1}{2} \sqrt{S}\left(\hat{x}_{a}-\hat{x}_{b}\right)-\sum_{i=1}^{n} \hat{p}_{T}^{(i)} \sinh \hat{\eta}_{i}-\hat{\alpha}_{T} \sinh \hat{\eta}_{q}\right) .
\end{aligned}
$$

After integrating $\hat{\vec{q}}_{T}, \hat{x}_{a}, \hat{x}_{b}$ over the Kronecker $\delta$-functions, we obtain

$$
d \hat{x}_{a} d \hat{x}_{b} d \Phi\left(\hat{p}_{a} \hat{p}_{b} ; \hat{Q},\{\hat{p}\}_{n}\right)=\frac{1}{\left(16 \pi^{3}\right)^{n+1}} \frac{2}{S}\left(\prod_{i=1}^{n} d \hat{p}_{T}^{(i)} d \hat{\eta}_{i} d \hat{\phi}_{i} \times \hat{p}_{T}^{(i)}\right) \times d \hat{\eta}_{q} \times \Theta\left(1-\hat{x}_{1}\right) \Theta\left(1-\hat{x}_{2}\right),
$$


with

$$
\begin{aligned}
& \hat{\vec{q}}_{T}=-\sum_{i=1}^{n} \hat{\bar{p}}_{T}^{(i)} \\
& \hat{x}_{a}=\frac{1}{\sqrt{S}}\left(\hat{\alpha}_{T} e^{\eta_{q}}+\sum_{i=1}^{n} \hat{p}_{T}^{(i)} e^{\hat{\eta}_{i}}\right) \\
& \hat{x}_{b}=\frac{1}{\sqrt{S}}\left(\hat{\alpha}_{T} e^{-\eta_{q}}+\sum_{i=1}^{n} \hat{p}_{T}^{(i)} e^{-\hat{\eta}_{i}}\right),
\end{aligned}
$$

and the constraints for a physical event, $\hat{x}_{a, b} \leq 1$, given by the $\Theta$-functions.

\subsection{The final state FBPS generator}

The final state generator begins with a $V+$ jet(s) Born event. One of the massless Born jets is split into two partons generating a massive jet. To project back onto the massless Born jet we scale the longitudinal momentum, $\vec{p}_{12}^{L}$ of the massive jet, leaving invariant both the transverse momentum and the rapidity of the jet

$$
\begin{aligned}
p_{a}+p_{b}-Q-p_{1}-p_{2}-P & =p_{a b}-Q-p_{12}-P \\
& =\left(p_{a b}+\alpha p_{12}^{L}\right)-Q-\left(p_{12}+\alpha p_{12}^{L}\right)-P \\
& =\hat{p}_{a b}-\hat{Q}-\hat{p}_{J}-\hat{P},
\end{aligned}
$$

where $\hat{Q}=Q, \hat{p}_{a b}=\hat{p}_{a}+\hat{p}_{b}=p_{a}+p_{b}+\alpha p_{12}^{L}, \hat{p}_{J}=p_{12}+\alpha p_{12}^{L}, p_{12}^{L}=\left(p_{1}+p_{2}\right)_{L}=$ $\left(0,0,\left(p_{1}\right)_{z}+\left(p_{2}\right)_{z}, E_{1}+E_{2}\right), P=\hat{P}=p_{3}+\cdots+p_{n}$ and $\alpha$ is given by the constraint $\hat{p}_{J}^{2}=0$. Note that $\hat{p}_{a}$ and $\hat{p}_{b}$ are reconstructed from $\hat{p}_{a b}$ using $\frac{1}{2} \sqrt{S} \times \hat{x}_{a, b}=\hat{E}_{a b} \pm\left(\hat{p}_{z}\right)_{a b}$ in the momenta reconstruction of eq. (2.3).

To derive the FBPS generator we first encode the branching in a decomposition of unity

$$
1=2 \sqrt{\left(p_{12}^{L}\right)^{2}\left(p_{12}^{T}\right)^{2}} \int d \alpha d \hat{p}_{J} \delta\left(\hat{p}_{J}^{2}\right) \delta\left(\hat{p}_{J}-p_{12}-\alpha p_{12}^{L}\right),
$$

insert it into the 3 -particle phase space generator

$$
d \Phi_{3}\left(p_{a}, p_{b} ; Q, p_{1}, p_{2}\right)=\frac{d Q}{(2 \pi)^{3}} \frac{d p_{1}}{(2 \pi)^{3}} \frac{d p_{2}}{(2 \pi)^{3}} \delta\left(Q^{2}-M_{V}^{2}\right) \delta\left(p_{1}^{2}\right) \delta\left(p_{2}^{2}\right) \delta\left(p_{a}+p_{b}-Q-p_{1}-p_{2}\right),
$$

and integrate $p_{2}$ over $\delta\left(\hat{p}_{J}-p_{12}-\alpha p_{12}^{L}\right)$ and subsequently integrate $\alpha$ over $\delta\left(p_{2}^{2}\right)$ to obtain the desired FBPS generator

$$
d \Phi_{3}^{\mathrm{FINAL}}\left(p_{a}, p_{b} ; Q, p_{1}, p_{2}\right)=d \Phi_{2}\left(\hat{p}_{a}, \hat{p}_{b} ; \hat{Q}, \hat{p}_{J}\right) \times\left[\frac{d p_{1}}{(2 \pi)^{3}} \delta\left(p_{1}^{2}\right)\right] \times J\left(\hat{p}_{J}, p_{1}\right) .
$$

The momenta are given by $p_{2}^{T}=\hat{p}_{J}^{T}-p_{1}^{T}, p_{2}^{L}=\beta_{+} \hat{p}_{J}^{L}-p_{1}^{L}, p_{a b}=\hat{p}_{a b}-\left(1-\beta_{+}\right) \hat{p}_{J}^{L}, Q=\hat{Q}$ and the Jacobian is given by

$$
J\left(\hat{p}_{J}, p_{1}\right)=\left|\frac{2}{1-\beta_{-} / \beta_{+}}\right| \times \sqrt{\frac{\left(\hat{p}_{J}^{T}\right)^{2}}{\left(\hat{p}_{J}^{L}\right)^{2}}}=\left|\frac{2}{1-\beta_{-} / \beta_{+}}\right|,
$$



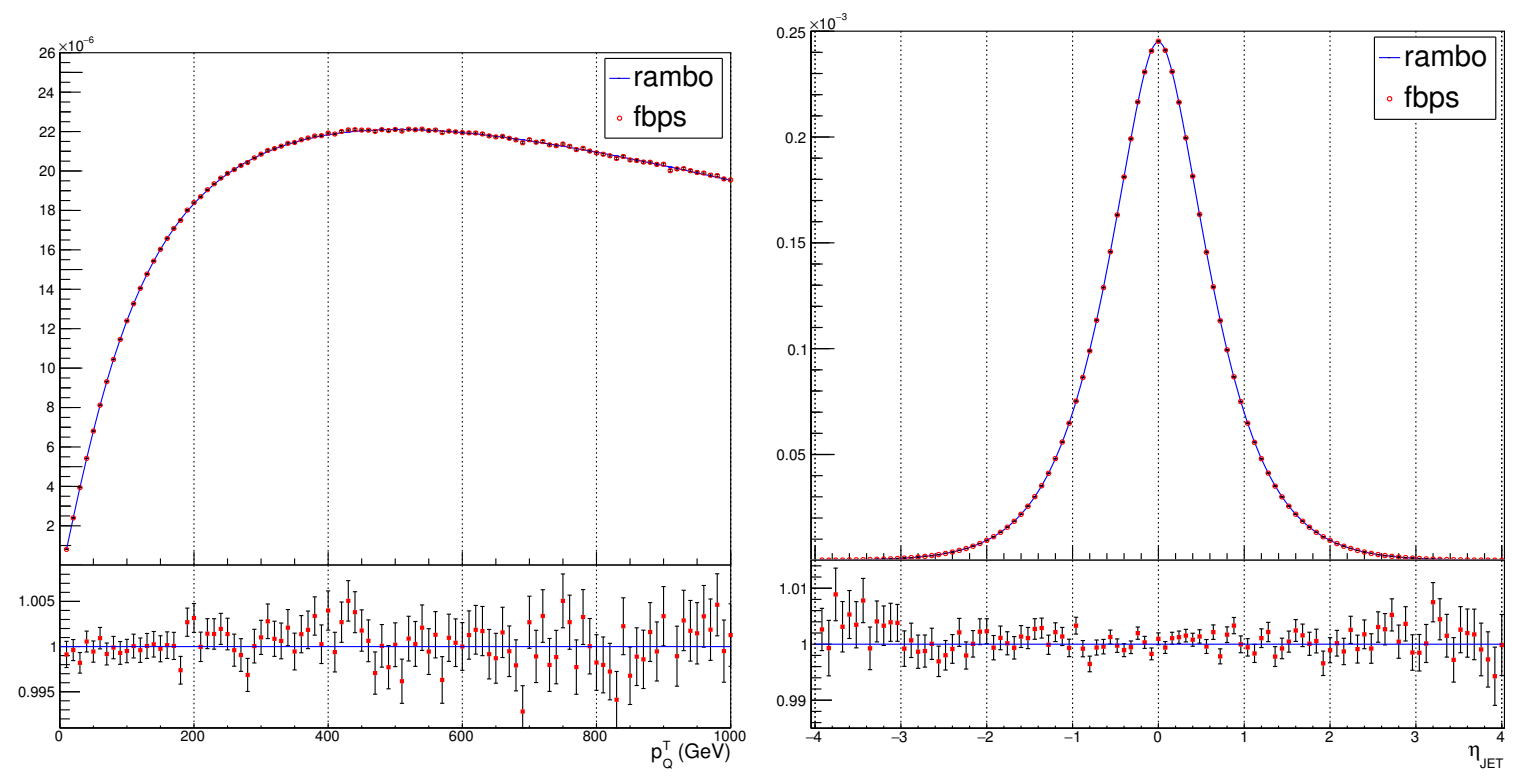

Figure 1. A comparison between RAMBO and the FBPS generator of eq. (2.10). The left graph compares the vector boson transverse momentum, while the right graph compares the jet rapidity. For Rambo the $V+2$ particle phase space was generated and a jet algorithm applied to obtain a $V+1$ jet inclusive final state. For the FBPS generator the observables are the Born momenta, while the phase space weight is re-weighted by the bremsstrahlung event(s).

where

$$
\beta_{ \pm}=\frac{\left(\hat{p}_{J}^{L} \cdot p_{1}^{L}\right) \pm \sqrt{\left(\hat{p}_{J}^{T}\right)^{4}+2\left(\hat{p}_{J}^{L}\right)^{2}\left(\hat{p}_{J}^{T} \cdot p_{1}^{T}\right)+\left(\hat{p}_{J}^{L} \cdot p_{1}^{L}\right)^{2}}}{\left(\hat{p}_{J}^{L}\right)^{2}}
$$

The upper limit on the integration over $p_{1}$ is determined by the condition on the parton fractions $x_{a, b}<1$. Because the bremsstrahlung is generated mostly soft and/or collinear with the jet momentum this condition can be implemented through a veto without any noticeable impact on the performance of the generator.

The generator is a complete phase space generator, however it is designed to be used in a different manner. This final state brancher is generated from a fixed Born event. By repeatedly branching this event, one integrates over the jet mass and obtains the final state part of the $K$-factor for this Born event. The FBPS generator of eq. (2.10) is compared to the RAMBO phase space generator [18] in figure 1.

\subsection{The initial state FBPS generator}

The initial state brancher is more complicated due to the fact that the extra parton generated is part of the beam jet and not added to the final state jets. As a consequence the transverse momentum generated by the branching momentum will have to be balanced by the vector boson or absorbed into the jet. At higher orders this generates events with transverse momentum of the vector boson below the transverse momentum cut on the jet transverse momentum. 
We first derive the generator which leaves the jet momentum $p_{J}$ invariant. The cluster map is given by

$$
\hat{p}_{a b}-\hat{Q}-\hat{p}_{J}=\left(\hat{p}_{a b}-\alpha p_{1}^{L}\right)-\left(\hat{Q}-\alpha p_{1}^{L}-p_{1}\right)-\hat{p}_{J}-p_{1}=p_{a b}-Q-p_{J}-p_{1},
$$

where $p_{J}=\hat{p}_{J}, p_{a b}=\hat{p}_{a b}-\alpha p_{1}^{L}, Q=\hat{Q}-\hat{p}_{1}^{T}-(1+\alpha) p_{1}^{L}$ and $\alpha$ is given by the constraint that $Q^{2}=\hat{Q}^{2}=M_{V}^{2}$.

The first step is again to encode the above branching in a partition of unity

$$
1=2 \sqrt{\left(p_{1}^{L}\right)^{4}-2\left(p_{1}^{L}\right)^{2}\left(p_{1}^{T} \cdot Q\right)+\left(p_{1}^{L} \cdot Q\right)^{2}} \int d \alpha d \hat{Q} \delta\left(\hat{Q}^{2}-M_{V}^{2}\right) \delta\left(\hat{Q}-Q-p_{1}^{T}-(1+\alpha) p_{1}^{L}\right),
$$

and insert it in the 3 particle phase space

$$
d \Phi_{3}\left(p_{a}, p_{b} ; Q, p_{J}, p_{1}\right)=\frac{d Q}{(2 \pi)^{3}} \frac{d p_{J}}{(2 \pi)^{3}} \frac{d p_{1}}{(2 \pi)^{3}} \delta\left(Q^{2}-M_{V}^{2}\right) \delta\left(p_{J}^{2}\right) \delta\left(p_{1}^{2}\right) \delta\left(p_{a}+p_{b}-Q-p_{J}-p_{1}\right) .
$$

By integrating $Q$ over $\delta\left(\hat{Q}-Q-p_{1}^{T}-(1+\alpha) p_{1}^{L}\right)$ and $\alpha$ over $\delta\left(Q^{2}-M_{V}^{2}\right)$ one obtains the FBPS generator

$$
d \Phi_{3}^{\mathrm{INIT,I}}\left(p_{a}, p_{b} ; Q, p_{J}, p_{1}\right)=d \Phi_{2}\left(\hat{p}_{a}, \hat{p}_{b} ; \hat{Q}, \hat{p}_{J}\right) \times\left[\frac{d p_{1}}{(2 \pi)^{3}} \delta\left(p_{1}^{2}\right)\right] \times J\left(\hat{Q}, p_{1}\right) .
$$

The momenta are given by $Q_{T}=\hat{Q}_{T}-p_{1}^{T}, Q_{L}=\hat{Q}_{L}-(1+\alpha) p_{1}^{L}, p_{a b}=\hat{p}_{a b}-\alpha p_{1}^{L}, p_{J}=\hat{p}_{J}$ and the Jacobian is given by

$$
J\left(\hat{Q}, p_{1}\right)=\sqrt{\frac{\left(p_{1}^{L}\right)^{4}-2\left(p_{1}^{L}\right)^{2}\left(p_{1}^{T} \cdot Q_{T}\right)+\left(p_{1}^{L} \cdot Q_{L}\right)^{2}}{\left(p_{1}^{L}\right)^{4}+2\left(p_{1}^{L}\right)^{2}\left(p_{1}^{T} \cdot \hat{Q}_{T}\right)+\left(\hat{p}_{1}^{L} \cdot \hat{Q}_{L}\right)^{2}}},
$$

where

$$
\alpha=\frac{\left(p_{1}^{L} \cdot \hat{Q}_{L}\right)-\left(p_{1}^{L}\right)^{2}-\sqrt{\left(p_{1}^{L}\right)^{4}+2\left(p_{1}^{L}\right)^{2}\left(p_{1}^{T} \cdot \hat{Q}_{T}\right)+\left(p_{1}^{L} \cdot \hat{Q}_{L}\right)^{2}}}{\left(p_{1}^{L}\right)^{2}} .
$$

Because the jet transverse momentum and rapidity is invariant under the branching the Born phase space can be generated with the given jet cuts. The comparisons with RAMBO are shown in figure 2 .

Next we construct the generator, leaving the vector boson momentum $Q$ invariant. The clustering is now given by

$$
\hat{p}_{a b}-\hat{Q}-\hat{p}_{J}=\left(\hat{p}_{a b}-\alpha p_{1}^{L}\right)-\hat{Q}-\left(\hat{p}_{J}-\alpha p_{1}^{L}-p_{1}\right)-p_{1}=p_{a b}-Q-p_{J}-p_{1},
$$

where $Q=\hat{Q}, p_{a b}=\hat{p}_{a b}-\alpha p_{1}^{L}, p_{J}=\hat{p}_{J}-p_{1}^{T}-(1+\alpha) p_{1}^{L}$ and $\alpha$ is given by the constraint that $p_{J}^{2}=\hat{p}_{J}^{2}$.

Encoding the above branching in a partition of unity

$$
1=2 \sqrt{\left(p_{1}^{L}\right)^{4}-2\left(p_{1}^{L}\right)^{2}\left(p_{1}^{T} \cdot p_{J}^{T}\right)+\left(p_{1}^{L} \cdot p_{J}^{L}\right)^{2}} \int d \alpha d \hat{p}_{J} \delta\left(\hat{p}_{J}^{2}\right) \delta\left(\hat{p}_{J}-p_{J}-p_{1}^{T}-(1+\alpha) p_{1}^{L}\right),
$$



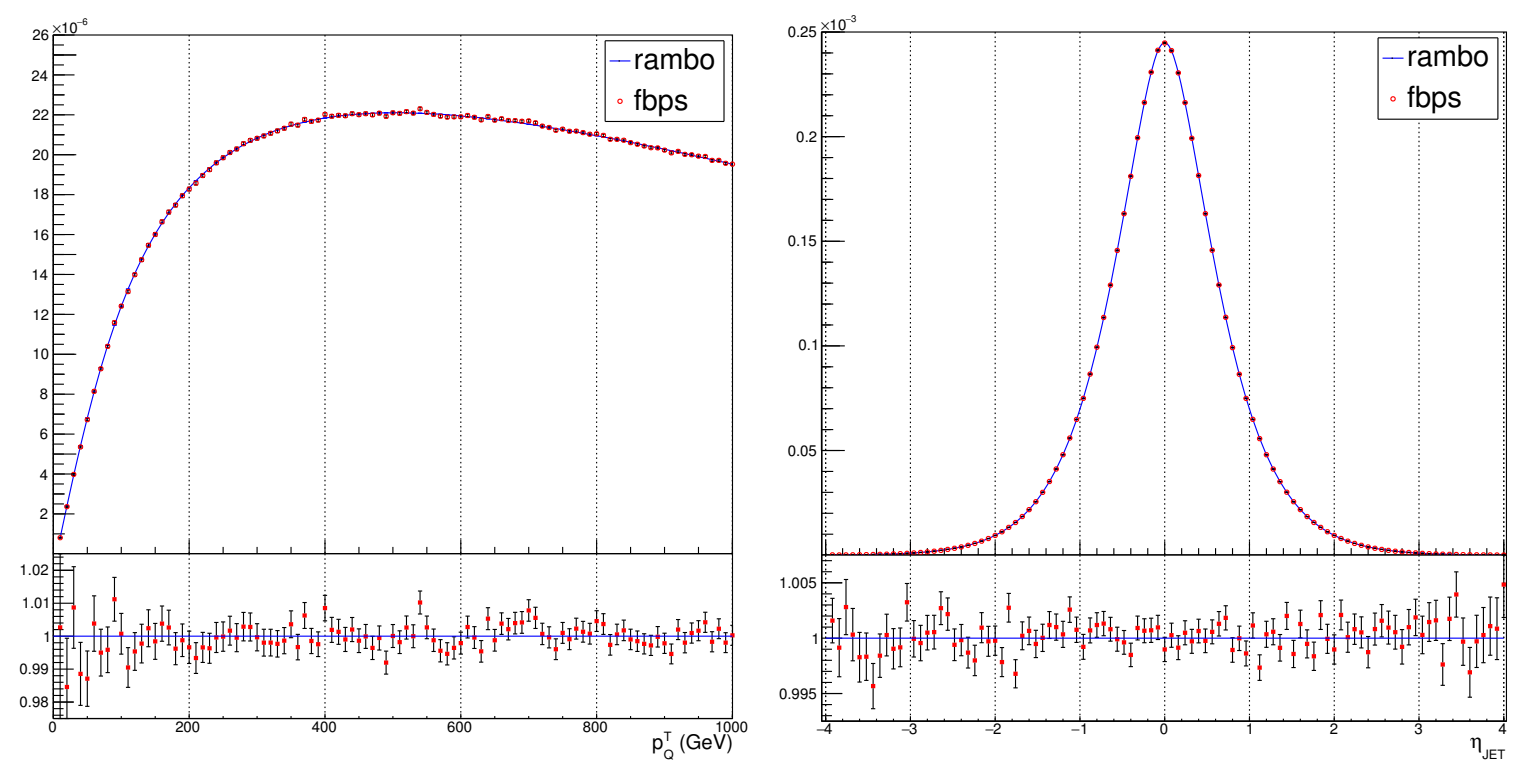

Figure 2. A comparison between RAMBO and the FBPS generator of eq. (2.16). The left graph compares the vector boson $p_{T}$, while the right graph compares the jet rapidity. For Rambo the $V+2$ particle phase space was generated and a jet algorithm applied to obtain a $V+1$ jet exclusive final state. For the FBPS generator the observables are the Born momenta, while the phase space weight is re-weighted by the bremsstrahlung event(s).

inserting it in the 3 -particle phase space,

$$
d \Phi_{3}\left(p_{a}, p_{b} ; Q, p_{J}, p_{1}\right)=\frac{d Q}{(2 \pi)^{3}} \frac{d p_{J}}{(2 \pi)^{3}} \frac{d p_{1}}{(2 \pi)^{3}} \delta\left(Q^{2}-M_{V}^{2}\right) \delta\left(p_{J}^{2}\right) \delta\left(p_{1}^{2}\right) \delta\left(p_{a}+p_{b}-Q-p_{J}-p_{1}\right)
$$

and subsequently integrating $p_{J}$ over $\delta\left(\hat{p}_{J}-p_{J}-p_{1}^{T}-(1+\alpha) p_{1}^{L}\right)$ and $\alpha$ over $\delta\left(p_{J}^{2}\right)$ one obtains

$$
d \Phi_{3}^{\mathrm{INIT}, \mathrm{II}}\left(p_{a}, p_{b} ; Q, p_{J}, p_{1}\right)=d \Phi_{2}\left(\hat{p}_{a}, \hat{p}_{b} ; \hat{Q}, \hat{p}_{J}\right) \times\left[\frac{d p_{1}}{(2 \pi)^{3}} \delta\left(p_{1}^{2}\right)\right] \times J\left(\hat{p}_{J}, p_{1}\right) .
$$

The momenta are given by $p_{J}^{T}=\hat{p}_{J}^{T}-p_{1}^{T}, p_{J}^{L}=\hat{p}_{J}^{L}-(1+\alpha) p_{1}^{L}, p_{a b}=\hat{p}_{a b}-\alpha p_{1}^{L}, Q=\hat{Q}$ and the Jacobian is given by

$$
J\left(\hat{p}_{J}, p_{1}\right)=\sqrt{\frac{\left(p_{1}^{L}\right)^{4}-2\left(p_{1}^{L}\right)^{2}\left(p_{1}^{T} \cdot p_{J}^{T}\right)+\left(p_{1}^{L} \cdot p_{J}^{L}\right)^{2}}{\left(p_{1}^{L}\right)^{4}+2\left(p_{1}^{L}\right)^{2}\left(p_{1}^{T} \cdot \hat{p}_{J}^{T}\right)+\left(p_{1}^{L} \cdot \hat{p}_{J}^{L}\right)^{2}}},
$$

where

$$
\alpha=\frac{\left(p_{1}^{L} \cdot \hat{p}_{J}^{L}\right)-\left(p_{1}^{L}\right)^{2}-\sqrt{\left(p_{1}^{L}\right)^{4}+2\left(p_{1}^{L}\right)^{2}\left(p_{1}^{T} \cdot \hat{p}_{J}^{T}\right)+\left(p_{1}^{L} \cdot \hat{p}_{J}^{L}\right)^{2}}}{\left(p_{1}^{L}\right)^{2}} .
$$

Because the jet gets a kick due to the emission of the extra parton, we cannot impose the jet cuts on the Born generator. The comparisons with RAMBO are shown in figure 3. 

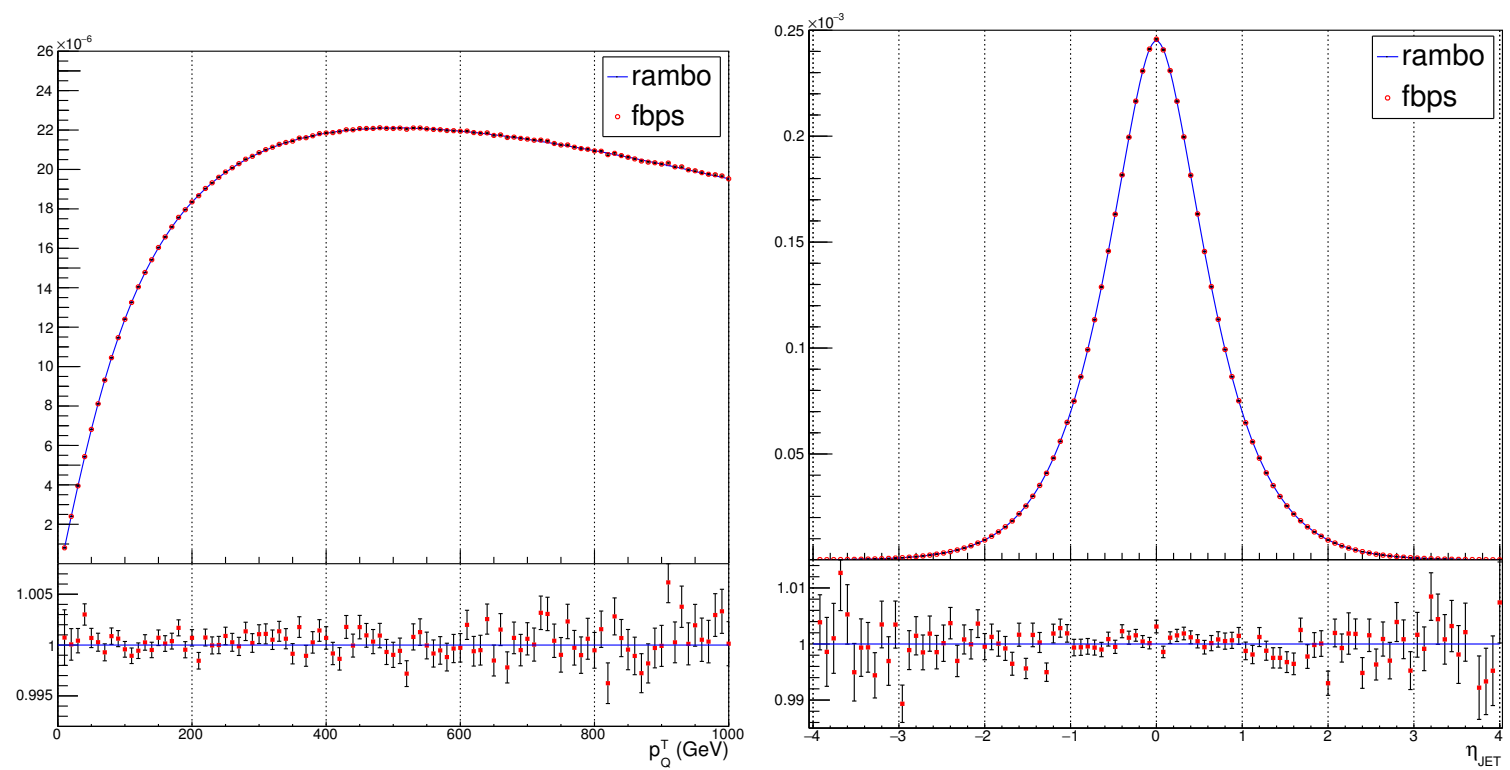

Figure 3. A comparison between RAMBO and the FBPS generator of eq. (2.22). The left graph compares the vector boson $p_{T}$, while the right graph compares the jet rapidity. For Rambo the $V+2$ particle phase space was generated and a jet algorithm applied to obtain a $V+1$ jet exclusive final state. For the FBPS generator the observables are the Born momenta, while the phase space weight is re-weighted by the bremsstrahlung event(s).

\subsection{Kinematics}

The above FBPS generators require us to generate the massless bremsstrahlung particle $p_{1}$. One method is to utilize the jettiness variables which are equivalent to normalized Sudakov parameters [19-22]. For the initial state brancher this is quite straightforward

$$
d p_{1} \delta\left(p_{1}^{2}\right)=\frac{1}{2} d p_{1}^{T} d \eta_{1} d \phi_{1} \times p_{1}^{T}=\frac{1}{4} d \tau_{a} d \tau_{b} d \phi_{1},
$$

with

$$
\tau_{a / b}=\left(p_{1} \cdot p_{a / b}\right) / E_{a / b}=p_{1}^{T} \exp \left( \pm \eta_{1}\right) .
$$

Given the integration variables $\tau_{a}, \tau_{b}$ and $\phi_{1}$ we can reconstruct the bremsstrahlung momentum

$$
p_{1}^{T}=\sqrt{\tau_{a} \tau_{b}} ; \eta_{1}=\frac{1}{2} \log \left(\frac{\tau_{a}}{\tau_{b}}\right) .
$$

This needs to be generalized to the final state brancher. To define the jettiness of the bremsstrahlung particle we choose the born jet momentum $\hat{p}_{J}$ and one of the born beam momenta, $\hat{p}_{a / b}$. Next, we apply a Sudakov decomposition [23] of the bremsstrahlung momentum $p_{1}$ with respect to $\hat{p}_{J}$ and $\hat{p}_{a / b}$

$$
p_{1}^{\mu}=\tau_{J} \frac{n_{1}^{\mu}}{\left(n_{1} \cdot n_{2}\right)}+\tau_{a / b} \frac{n_{2}^{\mu}}{\left(n_{1} \cdot n_{2}\right)}+p_{T}^{\mu},
$$

with

$$
n_{1}^{\mu}=\frac{\hat{p}_{J}^{\mu}}{\hat{E}_{J}} ; n_{2}^{\mu}=\frac{\hat{p}_{a / b}^{\mu}}{\hat{E}_{a / b}} ; n_{1}^{2}=n_{2}^{2}=\left(p_{T} \cdot n_{1 / 2}\right)=0 .
$$


By demanding that $p_{1}^{2}=0$ we find

$$
p_{T}^{2}=2 \frac{\tau_{J} \tau_{a / b}}{\left(n_{1} \cdot n_{2}\right)} ; \quad\left(p_{1} \cdot n_{1}\right)=\frac{\left(p_{1} \cdot \hat{p}_{J}\right)}{\hat{E}_{J}}=\tau_{J} ;\left(p_{1} \cdot n_{2}\right)=\frac{\left(p_{1} \cdot \hat{p}_{a / b}\right)}{\hat{E}_{a / b}}=\tau_{a / b} .
$$

To construct $p_{T}^{\mu}$ we choose two space-like momenta $n_{3 / 4}^{\mu}$ with the properties $\left(n_{1 / 2}\right.$. $\left.n_{3 / 4}\right)=0$ and $n_{3,4}^{2}=-1$, then

$$
p_{T}^{\mu}=\sqrt{p_{T}^{2}}\left(\sin \phi \times n_{3}^{\mu}+\cos \phi \times n_{4}^{\mu}\right) .
$$

One possible construction of $n_{3 / 4}^{\mu}$ is given by

$$
\hat{n}_{3}^{\mu}=\delta_{n_{1} n_{2} g_{3}}^{n_{1} n_{2} \mu} ; \hat{n}_{4}^{\mu}=\delta_{n_{1} n_{2} n_{3} g_{4}}^{n_{1} n_{2} n_{3} \mu} ; n_{3 / 4}^{\mu}=\frac{\hat{n}_{3 / 4}^{\mu}}{\sqrt{-\left(\hat{n}_{3 / 4} \cdot \hat{n}_{3 / 4}\right)}},
$$

where $g_{3 / 4}^{\mu}$ are arbitrary reference momenta. The integration measure is given by

$$
d p_{1} \delta\left(p_{1}^{2}\right)=d \tau_{J} d \tau_{a / b} d \phi
$$

\section{The phase space generator for $\mathrm{V}+$ jets cross sections}

The final step is to construct a cross section phase space generator. This requires combining the initial state and final state FBPS generators. The introduction of a jet algorithm will do this by partitioning the phase space into individual sectors, one for each parton. The phase space for the two sectors associated with the initial state partons will use an initial state FBPS generator. While each sector associated with a jet will use the final state FBPS generator.

The sectors are determined by the distance measures between the partons/clusters given by the jet algorithm. For the $k_{T}$-jet family of jet algorithms $[24,25]$ we have

$$
d_{i j}=\min \left(\left(p_{i}^{T}\right)^{2 p},\left(p_{j}^{T}\right)^{2 p}\right) \times\left(\frac{\Delta_{i j}^{2}}{R^{2}}\right) ; d_{i B}=\left(p_{i}^{T}\right)^{2 p},
$$

where

$$
\Delta_{i j}^{2}=\left(\eta_{i}-\eta_{j}\right)^{2}+\left(\phi_{i}-\phi_{j}\right)^{2} .
$$

For the example of $P P \rightarrow V+1$ jet we use the jet algorithm to separate the phase space into two initial state bremsstrahlung sectors and the final state bremsstrahlung sector, i.e., the 0 -jet, 1-jet, and 2-jet sectors. In this NLO example the jet algorithm simplifies significantly which in general will not be the case. In order to separate final state bremsstrahlung from other bremsstrahlung radiation, we use the decomposition of one to isolate the final state radiation

$$
1=\Theta\left(R-\Delta_{12}\right)+\Theta\left(\Delta_{12}-R\right) .
$$

However, the second term includes 2 -jet final state contributions. We can further decompose the second term

$1=\Theta\left(R-\Delta_{12}\right)+\Theta\left(\Delta_{12}-R\right) \times\left(\Theta\left(p_{\min }^{T}-p_{1}^{T}\right)+\Theta\left(p_{1}^{T}-p_{\min }^{T}\right)\right) \times\left(\Theta\left(p_{\min }^{T}-p_{2}^{T}\right)+\Theta\left(p_{2}^{T}-p_{\min }^{T}\right)\right)$. 

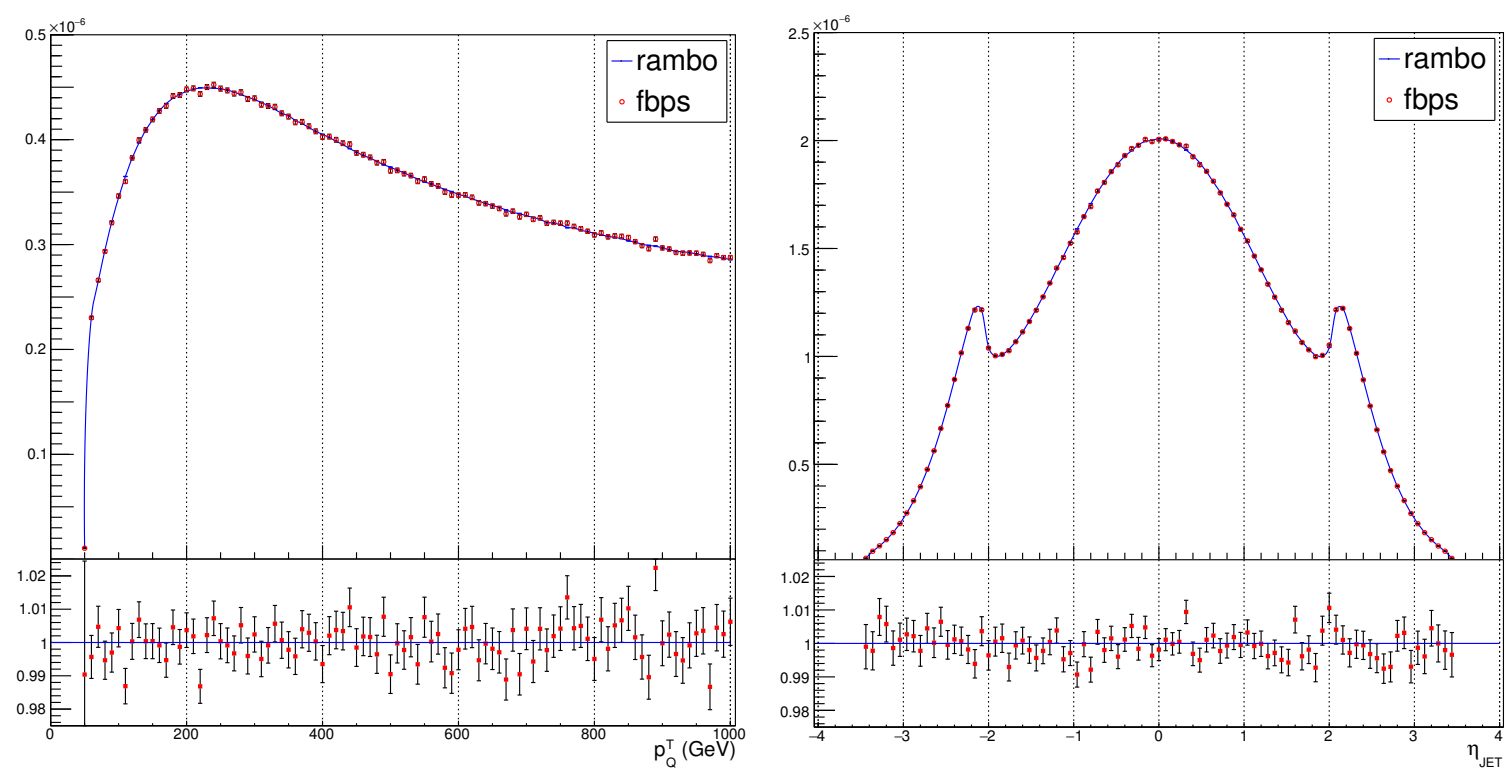

Figure 4. A comparison between RAMBO and the FBPS generator of eq. (3.6). The left graph compares the vector boson $p_{T}$, while the right graph compares the jet rapidity. For Rambo the $V+2$ particle phase space was generated and a jet algorithm applied to obtain a $V+1$ jet exclusive final state. For the FBPS generator the observables are the Born momenta, while the phase space weight is re-weighted by the bremsstrahlung event(s).

By requiring a 1-jet exclusive final state, we can filter out the 0-jet and 2-jet contributions, resulting in

$$
1=\Theta\left(R-\Delta_{12}\right)+\Theta\left(\Delta_{12}-R\right)\left(\Theta\left(p_{\min }^{T}-p_{1}^{T}\right) \Theta\left(p_{2}^{T}-p_{\min }^{T}\right)+\Theta\left(p_{\min }^{T}-p_{2}^{T}\right) \Theta\left(p_{1}^{T}-p_{\min }^{T}\right)\right)
$$

resulting in 3 sectors, one for each of the incoming beams and one for the final state jet. It is straightforward to generalize this procedure to multiple jet final states.

The $P P \rightarrow V+1$ exclusive jet phase space now becomes

$$
\begin{aligned}
& d \Phi_{3}^{\text {exclusive }}\left(p_{a}, p_{b} ; Q, p_{1}, p_{2}\right)=d \Phi_{3}\left(p_{a}, p_{b} ; Q, p_{1}, p_{2}\right) \\
& \quad \times\left[\Theta\left(R-\Delta_{12}\right)+\Theta\left(\Delta_{12}-R\right)\left(\Theta\left(p_{\min }^{T}-p_{1}^{T}\right) \Theta\left(p_{2}^{T}-p_{\min }^{T}\right)+\Theta\left(p_{\min }^{T}-p_{2}^{T}\right) \Theta\left(p_{1}^{T}-p_{\min }^{T}\right)\right)\right] \\
& =d \Phi_{2}\left(\hat{p}_{a}, \hat{p}_{b} ; \hat{Q}, \hat{p}_{J}\right) \times\left[\frac{d p_{1}}{(2 \pi)^{3}} \delta\left(p_{1}^{2}\right)\right] \\
& \times\left[\Theta\left(R-\Delta_{12}\right) J^{\mathrm{FINAL}}\left(\hat{p}_{J}, p_{1}\right) \delta\left(M^{\mathrm{FINAL}}\left(\{\hat{p}\}_{2} \rightarrow\{p\}_{2}\right)\right)\right. \\
& \left.+\Theta\left(\Delta_{12}-R\right)\left(\Theta\left(p_{1}^{T}<p_{\mathrm{MIN}}^{T}\right) \Theta\left(p_{2}^{T}>p_{\mathrm{MIN}}^{T}\right)+(1 \leftrightarrow 2)\right) J^{\mathrm{INIT}}\left(\hat{Q}, p_{1}\right) \delta\left(M^{\mathrm{INIT}}\left(\{\hat{p}\}_{2} \rightarrow\{p\}_{2}\right)\right)\right] .
\end{aligned}
$$

By adding the initial state transverse momentum kick to the vector boson we leave the jet momentum invariant. In figure 4 we show the validation of the cross section phase space generator in eq. (3.6) by comparing to the RAMBO phase space generator using a $k_{T}$ jet algorithm. To see the effect of initial state radiation we show in figure 5 the change in the vector boson momentum due to the initial state bremsstrahlung radiation. If one 

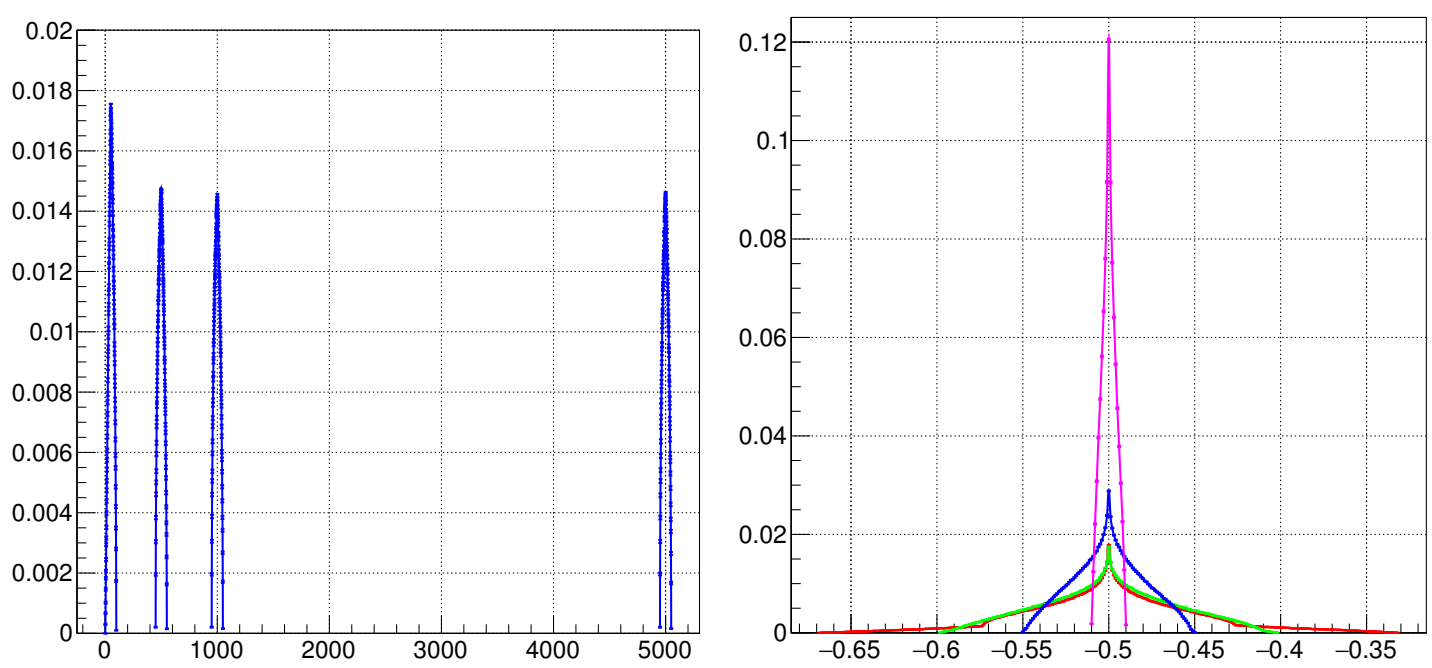

Figure 5. The transverse momentum (left) and the rapidity (right) of the vector boson after initial state radiating for a fixed jet momentum with transverse momentum $p_{T}^{\text {jet }}=50,500,1000,5000$ and rapidity of 0.4 and born rapidity of the vector boson equal to -0.5 .

chooses to add the unclustered partonic radiation back to the vector boson momentum one would recover fully the initiating Born event.

Alternatively, one could choose to give the initial state radiation transverse momentum kick to the jet, leaving the vector boson momentum invariant. Again by balancing the transverse momentum of the final state objects by adding the unclustered partonic transverse momentum to the jet momentum the originating Born event is fully reconstructed.

\section{Conclusions}

The use of a FBPS generator offers many improvements for higher order calculations compared to conventional phase space generators. These all stem from the property that the bremsstrahlung is generated from an originating Born event with the observables calculated from the Born event momenta. This feature provides us the ability to define single event weights beyond Leading Order.

From a numerical viewpoint, calculating the higher order corrections to the event weight is vastly improved as the dimensionality of the phase space integration at NLO is only three and independent of the jet multiplicity. This makes the integration tractable and straightforward to optimize with a large variety of numerical integration options available. Each colored line in the event has its own independent 3-dimensional integration. The cancellations between virtual and soft/collinear can be fine-tuned per Born event. One drawback of the method is that the subtraction method has to match this approach. One has to ensure the subtraction term projects back to the Born event and not to a variety of different Born events. The more physically motivated slicing methods will not be affected by this issue since these methods always project back to the originating Born event $[12,26]$.

In general the observable can be smeared by the bremsstrahlung, as is shown in figure 5. However, this smearing is per construction infrared unstable as it is generated by 
soft/collinear radiation pushing the observable away from its Born value. Using the Born event momenta to define the observable will guarantee an infrared insensitive observable and hence a stable prediction. Predictions like the jet mass are best made using shower Monte Carlo programs which can be easily matched to the fixed LO event to fill back in the opaque jet with hadrons and add the initial state hadronic radiation, providing a fully exclusive final state. This will set up a natural separation between the fixed order perturbative part and the shower part of the prediction, with higher order corrections providing a $K$-factor to the showered LO event.

One can go a step further and produce an unweighted LO event sample, shower/ hadronize the event and apply detector corrections. Next we can generate for each of these LO events a $K$-factor using the FBPS generator (see also ref. [8]). Assuming we have perturbative convergence, the $K$-factor should be of order one. This will re-weight the showered and detector corrected Born event with a weight around one. Applying radiative corrections in this manner is straightforward and time efficient. The experimental jet algorithm does not need to be changed as long as the observable is unaltered by the bremsstrahlung radiation, i.e. the most exclusive observable is given by $d \sigma /\left(d Q\left\{d \vec{p}_{T}^{i} d \eta_{i}\right\}_{i=1}^{n}\right)$ using the generator of eq. (3.6). One can go further and change the clustering phase of the jet algorithm itself to obtain massless jets and cluster the initial state radiation back into the final state objects. Then the exact Born event is reconstructed and any observable using the Born event momenta can be used to define observables.

The next phase of the project is to apply the FBPS generator to the NLO calculations of $P P \rightarrow V+1$ jet, $P P \rightarrow 2$ jets and $P P \rightarrow V+2$ jets. Each of these processes will add more complexity to the approach. After these processes have been successfully computed in the FBPS approach, we can begin investigating and constructing NNLO processes such as $P P \rightarrow V+1$ jet.

\section{Acknowledgments}

T.F. acknowledges the kind support of the Fermi Theory Group during several visits in 2017 and 2018. W.G. is supported by the DOE contract DE-AC02-07CH11359.

Open Access. This article is distributed under the terms of the Creative Commons Attribution License (CC-BY 4.0), which permits any use, distribution and reproduction in any medium, provided the original author(s) and source are credited.

\section{References}

[1] W.T. Giele, G.C. Stavenga and J.-C. Winter, A Forward Branching Phase-Space Generator, arXiv:1106.5045 [INSPIRE].

[2] J.M. Campbell, W.T. Giele and C. Williams, The Matrix Element Method at Next-to-Leading Order, JHEP 11 (2012) 043 [arXiv: 1204.4424] [INSPIRE].

[3] M. Cacciari, F.A. Dreyer, A. Karlberg, G.P. Salam and G. Zanderighi, Fully Differential Vector-Boson-Fusion Higgs Production at Next-to-Next-to-Leading Order, Phys. Rev. Lett. 115 (2015) 082002 [Erratum ibid. 120 (2018) 139901] [arXiv: 1506.02660] [INSPIRE]. 
[4] W.T. Giele, Improved Partonic Event Generators at Lepton Colliders, arXiv:1504.02137 [INSPIRE].

[5] J. Currie, T. Gehrmann, E.W.N. Glover, A. Huss, J. Niehues and A. Vogt, $N^{3} L O$ corrections to jet production in deep inelastic scattering using the Projection-to-Born method, JHEP 05 (2018) 209 [arXiv: 1803.09973] [INSPIRE].

[6] T. Martini and P. Uwer, Extending the Matrix Element Method beyond the Born approximation: Calculating event weights at next-to-leading order accuracy, JHEP 09 (2015) 083 [arXiv: 1506. 08798] [INSPIRE].

[7] T. Martini and P. Uwer, The Matrix Element Method at next-to-leading order QCD for hadronic collisions: Single top-quark production at the LHC as an example application, JHEP 05 (2018) 141 [arXiv:1712.04527] [INSPIRE].

[8] T. Martini, The Matrix Element Method at next-to-leading order QCD using the example of single top-quark production at the LHC, Ph.D. Thesis, Humboldt U., Berlin (2018) [DOI:10.18452/19288] [arXiv: 1807.06859] [INSPIRE].

[9] G.P. Lepage, A New Algorithm for Adaptive Multidimensional Integration, J. Comput. Phys. 27 (1978) 192 [inSPIRE].

[10] C.-H. Chung and T. Robens, Nagy-Soper subtraction scheme for multiparton final states, Phys. Rev. D 87 (2013) 074032 [arXiv:1209.1569] [InSPIRE].

[11] D. Goetz, C. Schwan and S. Weinzierl, Random Polarisations of the Dipoles, Phys. Rev. D 85 (2012) 116011 [arXiv: 1205.4109] [INSPIRE].

[12] T.O. Eynck, E. Laenen, L. Phaf and S. Weinzierl, Comparison of phase space slicing and dipole subtraction methods for $\gamma^{*} \rightarrow Q \bar{Q}$, Eur. Phys. J. C 23 (2002) 259 [hep-ph/0109246] [INSPIRE].

[13] S. Catani and M.H. Seymour, A General algorithm for calculating jet cross-sections in NLO QCD, Nucl. Phys. B 485 (1997) 291 [Erratum ibid. B 510 (1998) 503] [hep-ph/9605323] [INSPIRE].

[14] S. Frixione and B.R. Webber, Matching NLO QCD computations and parton shower simulations, JHEP 06 (2002) 029 [hep-ph/0204244] [INSPIRE].

[15] S. Frixione, P. Nason and C. Oleari, Matching NLO QCD computations with Parton Shower simulations: the POWHEG method, JHEP 11 (2007) 070 [arXiv: 0709. 2092] [INSPIRE].

[16] G. Corcella and M.H. Seymour, Initial state radiation in simulations of vector boson production at hadron colliders, Nucl. Phys. B 565 (2000) 227 [hep-ph/9908388] [INSPIRE].

[17] A. Buckley et al., General-purpose event generators for LHC physics, Phys. Rept. 504 (2011) 145 [arXiv: 1101.2599$]$ [INSPIRE].

[18] R. Kleiss, W.J. Stirling and S.D. Ellis, A New Monte Carlo Treatment of Multiparticle Phase Space at High-energies, Comput. Phys. Commun. 40 (1986) 359 [INSPIRE].

[19] I.W. Stewart, F.J. Tackmann and W.J. Waalewijn, N-Jettiness: An Inclusive Event Shape to Veto Jets, Phys. Rev. Lett. 105 (2010) 092002 [arXiv: 1004.2489] [InSPIRE].

[20] R. Boughezal, X. Liu and F. Petriello, $N$-jettiness soft function at next-to-next-to-leading order, Phys. Rev. D 91 (2015) 094035 [arXiv:1504.02540] [INSPIRE].

[21] J.M. Campbell, R.K. Ellis, R. Mondini and C. Williams, The NNLO QCD soft function for 1-jettiness, Eur. Phys. J. C 78 (2018) 234 [arXiv:1711.09984] [INSPIRE]. 
[22] J. Campbell, J. Huston and F. Krauss, The black book of quantum chromodynamics: a primer for the LHC era, Oxford University Press, Oxford (2018).

[23] V.V. Sudakov, Vertex parts at very high-energies in quantum electrodynamics, Sov. Phys. JETP 3 (1956) 65 [INSPIRE].

[24] S. Catani, Y.L. Dokshitzer, M.H. Seymour and B.R. Webber, Longitudinally invariant $K_{t}$ clustering algorithms for hadron hadron collisions, Nucl. Phys. B 406 (1993) 187 [INSPIRE].

[25] M. Cacciari, G.P. Salam and G. Soyez, The anti-k $k_{t}$ jet clustering algorithm, JHEP 04 (2008) 063 [arXiv: 0802.1189] [INSPIRE].

[26] B.W. Harris and J.F. Owens, The Two cutoff phase space slicing method, Phys. Rev. D 65 (2002) 094032 [hep-ph/0102128] [INSPIRE]. 